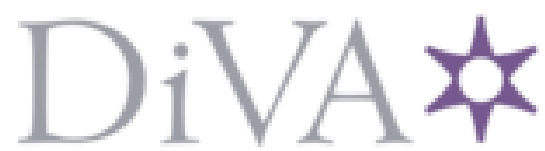

http://www.diva-portal.org

\title{
Postprint
}

This is the accepted version of a paper presented at 13th International Conference on the European Energy Market (EEM), JUN 06-09, 2016, Porto, PORTUGAL.

Citation for the original published paper:

Dimoulkas, I., Amelin, M., Hesamzadeh, M R. (2016)

Forecasting Balancing Market Prices Using Hidden Markov Models.

In: 201613 TH INTERNATIONAL CONFERENCE ON THE EUROPEAN ENERGY MARKET

(EEM) IEEE conference proceedings

International Conference on the European Energy Market

https://doi.org/10.1109/EEM.2016.7521229

N.B. When citing this work, cite the original published paper.

Permanent link to this version:

http://urn.kb.se/resolve?urn=urn:nbn:se:kth:diva-193848 


\section{Forecasting Balancing Market Prices Using Hidden Markov Models}

\author{
Ilias Dimoulkas \\ School of Electrical Engineering \\ Electric Power and Energy Systems \\ KTH Royal Institute of Technology \\ Stockholm, Sweden \\ Email: iliasd@kth.se
}

\author{
Mikael Amelin \\ School of Electrical Engineering \\ Electric Power and Energy Systems \\ KTH Royal Institute of Technology \\ Stockholm, Sweden \\ Email: amelin@kth.se
}

\author{
Mohammad Reza Hesamzadeh \\ Electricity Market Research Group \\ Electric Power and Energy Systems \\ KTH Royal Institute of Technology \\ Stockholm, Sweden \\ Email: mrhesamzadeh@ee.kth.se
}

\begin{abstract}
This paper presents a Hidden Markov Model (HMM) based method to predict the prices and trading volumes in the electricity balancing markets. The HMM are quite powerful in modelling stochastic processes where the underlying dynamics are not apparent. The proposed method provides both one hour and 12-36 hour ahead forecasts. The first is mostly useful to wind/solar producers in order to compensate their production imbalances while the second is important when submitting the offers to the day ahead markets. The results are compared to the ones from Markov-autoregressive model.
\end{abstract}

\section{INTRODUCTION}

Power generation from renewable energy sources (RES) is steadily increasing around the globe as many countries adopt policies that promote their use. In Europe for example, a target has been set that $20 \%$ of total energy consumption should be generated by RES till the year 2020 [1]. This will result in a RES share of $50 \%$ in power generation, which is going to be even higher in the future as new restrictions are applied regarding the $\mathrm{CO}_{2}$ emissions. The challenge of such high RES penetration is how the fluctuations in power production are going to be balanced. It is straightforward that the need for balancing power will increase which in turn will affect the balancing or real-time markets (BM), both in terms of the price volatility and the energy volume traded in them. Under these conditions, it is very crucial for power producers that are providing balancing power to estimate the prices in the BM in order to optimally schedule their production and balancing offers. The same applies for RES producers and power consumers who try to decrease their imbalance costs.

The field of price forecasting in the day ahead (DA) electricity markets has been well studied and new approaches are continuously proposed. Many different methods have been applied so far, ranging from econometric tools to pattern recognition and machine learning techniques [2]. On the other hand, the BM price modelling is not so well studied. It seems that the low liquidity in most of the BMs and the small number of participants affects the research interest for this type of electricity market. Among the methods that have been proposed for modelling and forecasting electricity prices in the BM, we can find autoregressive models [3]-[5], autoregressive models in combination with Markov processes
[6], non-linear time series models [7] and moving weighted average methods [3]. A benchmark of various BM forecasting models is provided in [8].

In this paper, we propose a method for BM electricity price and volume forecasting based on hidden Markov models (HMM). The HMM are used to model Markov processes with unobserved (hidden) states. Historically, the HMM has been used with success as predictor or classifier in many fields, like in speech recognition [9] and DNA sequence analysis [10]. Forecasting techniques based on HMM have been developed for the stock markets like in [11] and [12]. A HMM for DA electricity price forecasting has been used in [13] and [14].

The following sections are organised as follows: Section II describes the HMM. Section III explains how a HMM is trained and used for BM forecasting. In section IV a simulation is conducted with data coming from the Nordic BM. The results are presented and compared with a reference model. Finally, section V concludes this paper.

\section{THE HIDDEN MARKOV MODEL}

As it was referred in the introduction, a HMM can be used for modelling Markov processes with unobserved or hidden states. While in a regular Markov process the system moves between known states, in a HMM, the states are not directly visible (hidden), but the output (observation) which is dependent on the state, is visible. This is shown in fig. 1 where the hidden state at time $t$ is denoted as $x(t)$ and the observation at the same time as $y(t)$. As a Markov process, $x(t)$ is only dependent on $x(t-1)$ while $y(t)$ is only dependent on $x(t)$.

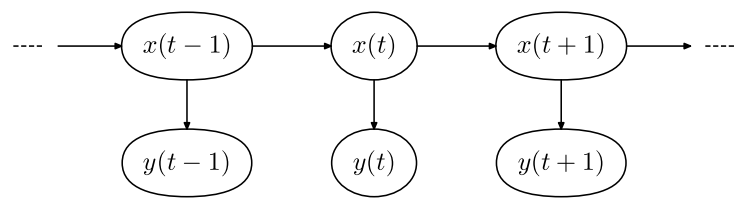

Fig. 1. A stochastic process modelled as a HMM with states $x$ and observations $y$

A HMM is defined by the following five elements [15]: 
1) A set of $N$ states $S=\left\{s_{1}, \ldots, s_{N}\right\}$ which are hidden. $x_{t}=s_{i}$ means that HMM is operating in state $s_{i}$ at time $t$. The hidden state sequence is denoted $X=$ $\left\{x_{1}, \ldots, x_{t}\right\}$.

2) A set of $M$ discrete observations $V=\left\{v_{1}, \ldots, v_{M}\right\}$. $y_{t}=v_{k}$ means that HMM output is $v_{k}$ at time $t$. The observation sequence is denoted $Y=\left\{y_{1}, \ldots, y_{t}\right\}$.

3) The state transition probability distribution matrix $A=$ $\left\{a_{i j}\right\}$, where $a_{i j}=P\left\{x_{t+1}=s_{j} \mid x_{t}=s_{i}\right\}, 1 \leq i, j \leq$ $N$ is the transition probability from state $s_{i}$ to state $s_{j}$. This implies that $a_{i j} \geq 0$ and $\sum_{j=1}^{N} a_{i j}=1,1 \leq i \leq N$.

4) The observation symbol probability distribution for each state $B=\left\{b_{j}(k)\right\}$, where $b_{j}(k)=$ $P\left\{y_{t}=v_{k} \mid x_{t}=s_{j}\right\}, 1 \leq j \leq N, 1 \leq k \leq M$ is the probability that observation $v_{k}$ is emitted in stage $s_{j}$. This implies that $b_{j}(k) \geq 0$ and $\sum_{k=1}^{M} b_{j}(k)=1,1 \leq j \leq N$.

5) The initial state probability distribution matrix $\pi=$ $\left\{\pi_{i}\right\}$, where $\pi_{i}=P\left\{x_{1}=s_{i}\right\}, 1 \leq i \leq N$ is the probability that the hidden state $s_{i}$ is the initial state.

When the observation are continuous like for example the values of the electricity prices, then the observation set $M$ is infinite and the observation symbol probability distribution cannot be calculated. In this case a continuous probability density function is used which is usually approximated by a mixture of Gaussian distributions $\mathrm{N}$ : $b_{j}\left(y_{t}\right)=$ $\sum_{m=1}^{M} c_{j m} N\left(\mu_{j m}, \Sigma_{j m}, y_{t}\right)$, where $M$ is the number of the mixtures and $c_{j m}, \mu_{j m}, \Sigma_{j m}$ are the mixture coefficient, the mean vector and the covariance matrix for the $\mathrm{m}$-th mixture in state $s_{j}$ respectively.

The notation $\lambda=(A, B, \pi)$ is used to define a HMM with discrete observations while $\lambda=\left(A, c_{j m}, \mu_{j m}, \Sigma_{j m}, \pi\right)$ for the continuous case.

\section{USING HMM FOR BALANCING MARKET FORECASTING}

A BM serves as a trading floor for replacement/tertiary reserves according to the ENTSO-E definition [16]. Using the BM, a power system operator (TSO/ISO) can ensure the balance of the system by activating balancing power offers usually submitted by conventional plants.

This paper is focusing on the Nordic BM, but the methodology described here can be also applied to other BMs with some minor modifications. The Nordic BM is characterized by a) marginal price settlement: price is set by the marginal cost of the last activated unit, b) hourly settlement resolution, c) dual imbalance pricing scheme: BM price is paid only to the balance responsible players that actively help the restoration of the system balance and d) price cap/floor: DA market prices act as an upper/lower limit to the BM prices [8]. There are three main possible balancing states depending on the direction of the balancing power needed each hour: 1) no regulation state, when consumption equals production, 2) upward regulation state, when consumption exceeds production, and 3) downward regulation state, when production exceeds consumption. There is also a fourth state which happens rather rarely and it is when both upward and downward regulation is needed for a specific hour.

Two prices are defined for each hour in the BM, the upward balancing price $\rho_{u p}$ and the downward balancing price $\rho_{\text {down }}$. Similarly, two trading power volumes are defined, the upward volume $V_{u p}$ and the downward volume $V_{\text {down }}$. The values that the $\mathrm{BM}$ prices and volumes can take depend on the balancing state as it shown in table I.

TABLE I

BALANCING VOLUMES AND PRICES RELATED TO THE BALANCING STATE

\begin{tabular}{|c|c|c|}
\hline States & BM volumes & BM prices \\
\hline \hline No regulation & $V_{u p}, V_{\text {down }}=0$ & $\rho_{u p}, \rho_{\text {down }}=\rho_{\text {spot }}$ \\
\hline Up reg. & $V_{u p}>0, V_{\text {down }}=0$ & $\rho_{u p} \geq \rho_{\text {spot }}, \rho_{\text {down }}=\rho_{\text {spot }}$ \\
\hline Down reg. & $V_{u p}=0, V_{\text {down }}<0$ & $\rho_{\text {down }} \leq \rho_{\text {spot }}, \rho_{\text {up }}=\rho_{\text {spot }}$ \\
\hline Up/Down reg. & $V_{u p}>0, V_{\text {down }}>0$ & $\rho_{u p} \geq \rho_{\text {sp. }}, \rho_{\text {down }} \leq \rho_{\text {sp. }}$. \\
\hline
\end{tabular}

It is more common to work with the BM premium instead of the BM prices directly. The BM premium is defined as (1):

$$
\delta_{u p / \text { down }}=\rho_{u p / \text { down }}-\rho_{\text {spot }}
$$

By definition, the BM upward premium $\delta_{u p}$ is positive or zero while the BM downward premium $\delta_{\text {down }}$ is negative or zero. The BM premiums for SE2 region and the year 2014 [17] are depicted in fig. 2. The same figure also depicts the power volumes for the same period.
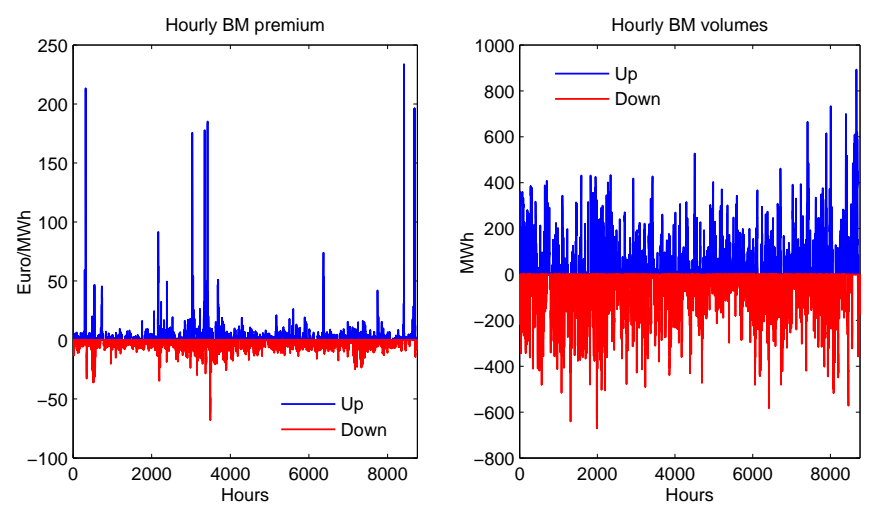

Fig. 2. BM premiums (left) and BM volumes (right) in SE2, 2014

The autocorrelation (ACF) and partial autocorrelation (PACF) functions of the BM premiums in fig. 3 show some strong correlation for lags 1-2. There is also some small correlation for lag 24, which implies a weak diurnal seasonality. This seasonality is stronger for downward regulation. BM volumes present similar autocorrelations. Furthermore, the scatter plot of the BM premiums against the BM volumes in fig. 4 shows some positive correlation between these two variables.

If a power producer participates both in the DA and balancing markets, the BM should be taken into account when submitting the offers to the DA market. In order to do that, the BM forecasting models should predict the prices/volumes for the next day, or 12-36 hours ahead as the bidding to 

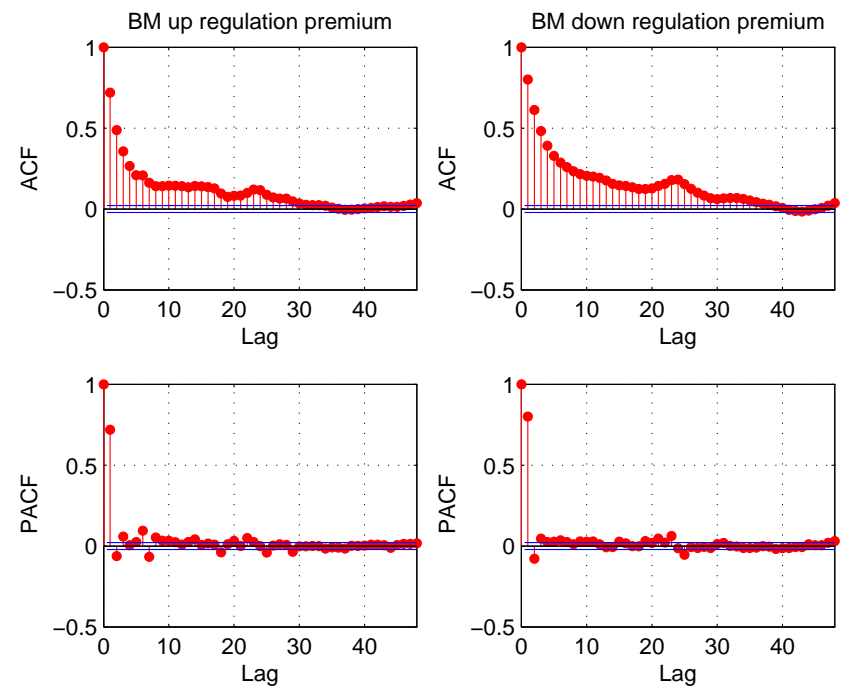

Fig. 3. ACF and PACF functions of BM premiums in SE2, 2014

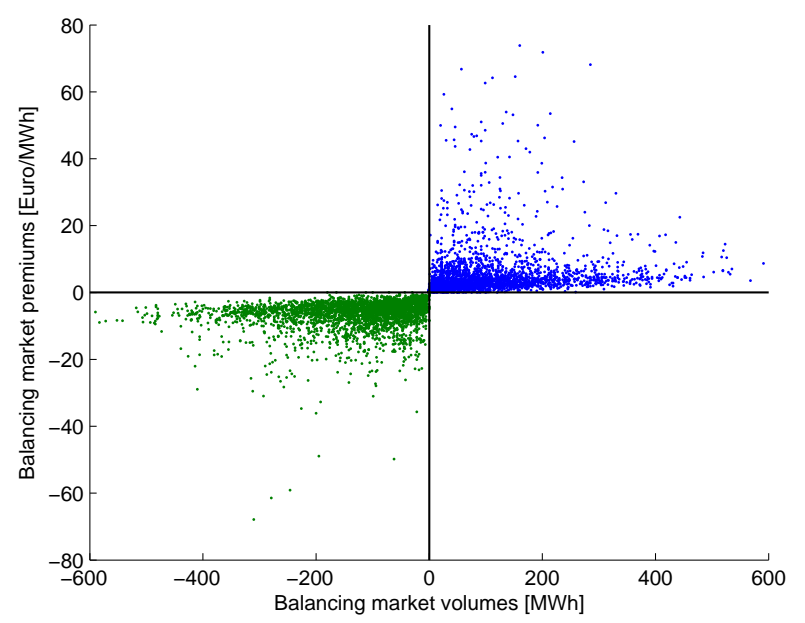

Fig. 4. Correlation between BM volumes and BM premiums in SE2, 2014

the DA market is typically done at noon of the previous day. Furthermore, RES producers that want to balance their stochastic production, are more interested for one hour ahead $\mathrm{BM}$ forecasts, because the production forecast error is lower closer to the real time production.

The proposed method can be used for both 1 hour and 12-36 hours ahead forecasts and it can be analysed in three steps:

1) Data preprocessing: It includes the outlier detection, the handling of the daylight saving time and the scaling of the data.

2) HMM training: During the training procedure the parameters of a HMM model $\lambda=\left(A, c_{j m}, \mu_{j m}, \Sigma_{j m}, \pi\right)$ are adjusted in a way to maximize $p\{Y, \lambda\}$ which is the probability that the given observation sequence $Y$ is generated by model $\lambda$. This is called the learning problem in HMM theory. Baum-Welch algorithm is used to train the model [15]. Here, two models are trained, one for up regulation with training vector $Y=\left[\delta_{u p} V_{u p}\right]$ and one for down regulation with training vector $Y=$ $\left[\delta_{\text {down }} V_{\text {down }}\right]$.

\section{3) Forecasting}

a) One hour ahead forecast: The Viterbi algorithm [15] is used to calculate the most probable state of the last hour in the training series which is the current hour. This is called the decoding problem in HMM theory. Then a marginalization of all possible cases for next hour state is done using eq. (2) where it is assumed that the current state $x_{t}=s_{i}$.

$$
y_{t+1}=\sum_{j=1}^{N} a_{i, j} \sum_{m=1}^{M} c_{j m} \mu_{j m}
$$

Eq. (2) generates non zero results for all the possible states $s_{i}$. This creates some error when there is no regulation. In order to decrease this error, the lowest possible output of eq. (2) is set to be zero.

b) Day ahead forecast: A different approach is used. Because it is very difficult to predict the states of the BM market for many hours ahead, the approach proposed is based on finding similar patterns in the past. For this, the forward-backward algorithm is used [15]. This procedure is called the evaluation problem in HMM theory. The algorithm calculates the likelihood of 24 hours observation sequences. Then the day $D_{N}$ which has the closest likelihood with the current day $D_{C}$ is chosen and the day that follows it $D_{N+1}$, is assumed to give the prediction for the day ahead. Alternatively, the differences of the two days $D_{N+1}$ and $D_{N}$ can be added to the current day $D_{C}$ for the day ahead prediction.

\section{Simulation}

This section presents the results of the proposed method. The data are collected from Nordpool [17] and specifically for the SE2 price area for the period 01.01.2014-31.12.2014. The data set is split into two sets: one training set and one validation set. The forecasting procedure is as follows: Data in the training set are used to train the model according to the procedure explained in the previous section. Then the trained model is used to forecast the BM premiums and volumes for the next hour (one hour ahead forecast) or for the next 36 hours (day ahead forecast). These forecasts are tested against the actual data included in the validation set and both the Mean Absolute Error (MAE) given by eq. (3) and the Symmetric Mean Absolute Percent Error (SMAPE) given by eq. (4) are calculated. SMAPE is a measure of prediction accuracy which is used instead of the commonly used Mean Absolute Percent Error (MAPE) because many of the actual values of the BM prices and volumes are zero. 


$$
\begin{gathered}
M A E=\frac{1}{N} \sum_{t=1}^{N}\left|F_{t}-A_{t}\right| \\
S M A P E=\frac{\sum_{t=1}^{N}\left|F_{t}-A_{t}\right|}{\sum_{t=1}^{N}\left(F_{t}+A_{t}\right)}
\end{gathered}
$$

Here $F_{t}$ is the forecasts, $A_{t}$ is the actual values and $N$ is the length of the data series.

Furthermore, in order to check if the data recentness affects the model accuracy, a moving window approach is used. According to this, the size of the training set is kept fixed and the earlier data are discarded. The size of the window which gives better results is decided after testing. With the updated training set, a new cycle starts with retraining of the model and forecast of the subsequent hour or day ahead. This procedure of the moving window is shown in fig. 5 .

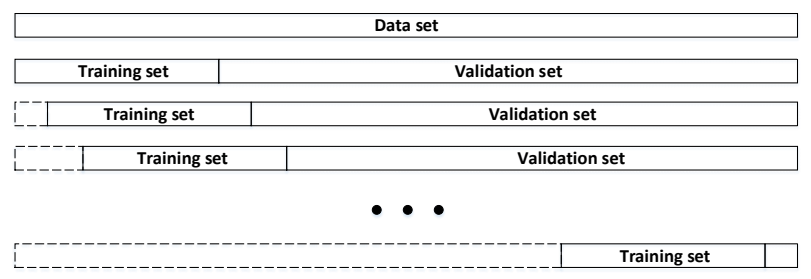

Fig. 5. The moving window concept for the training-forecasting procedure

\section{A. The reference model}

A reference model is built to compare the accuracy of the HMM method with it. This is a highly cited model [6] which uses a combination of a Markov switching state model to predict the state of the BM and a SARIMA model to predict the value of the BM prices. Here a modification of this model is used which combines elements of the models presented in [18] and [8].

The Markov switching model determines the balancing state among the four different states as shown in table I. A duration-dependent model is used with two transition matrices according to [6]. These transition matrices are given in table II.

TABLE II

TRANSITION MATRICES FOR THE DURATION-DEPENDENT MARKOV MODEL

\begin{tabular}{|c|c|c|c|c|c|c|c|c|c|}
\hline$p_{i j}^{1}$ & {$[1]$} & {$[2]$} & {$[3]$} & {$[4]$} & $p_{i j}^{2}$ & {$[1]$} & {$[2]$} & {$[3]$} & {$[4]$} \\
\hline \hline$[1]$ & 0.67 & 0.17 & 0.16 & 0 \\
\hline$[1]$ & 0.69 & 0.16 & 0.15 & 0 \\
\hline$[2]$ & 0.10 & 0.84 & 0.03 & 0.02 \\
\hline$[3]$ & 0.19 & 0.04 & 0.79 & 0.02 \\
\hline$[2]$ & 0.11 & 0.83 & 0.03 & 0.02 \\
\hline$[4]$ & 0.07 & 0.36 & 0.54 & 0.03 \\
\hline$[3]$ & 0.16 & 0.05 & 0.77 & 0.02 \\
\hline$[4]$ & 0 & 1 & 0 & 0 \\
\hline
\end{tabular}

(a) Transition probabilities for moving(b) Transition probabilities for moving to state $j$ after being in state $i$ for oneto state $j$ after being in state $i$ for two hour
The BM continuous valued prices and volumes are modelled with ARIMA family models. An $\operatorname{ARMA}(1,1)$ is found to best fit the $\mathrm{BM}$ up regulation volumes while an $\operatorname{ARMA}(2,1)$ is the best for the BM down regulation volumes. Regarding the BM premiums, an $\operatorname{ARMA}(1,1)$ is also found to best fit the BM up regulation premiums while an $\operatorname{ARMA}(2,1)$ or $\operatorname{ARMAX}(2,1)$ are suitable for the $\mathrm{BM}$ down regulation premiums. The external predictor in the case of the ARMAX model is the volume forecast. The parameters of the four models are given in table III. $\Phi_{p}, \Theta_{p}$ are the autoregressive and moving average parameters for order $\mathrm{p}$ while $\sigma^{2}$ is the variance and $c$ the constant.

TABLE III

ARMA MODEL PARAMETERS

\begin{tabular}{|c|c|c|c|c|c|}
\cline { 2 - 6 } \multicolumn{1}{c|}{} & $\Phi_{1}$ & $\Phi_{2}$ & $\Theta_{1}$ & $\sigma^{2}$ & $c$ \\
\hline$V_{\text {up }}$ & 0.66 & - & 0.21 & 1847 & 10.2 \\
\hline$V_{\text {down }}$ & 0.49 & 0.18 & 0.43 & 2962 & -17.7 \\
\hline$\delta_{\text {up }}$ & 0.61 & - & 0.15 & 38.2 & 0.81 \\
\hline$\delta_{\text {down }}$ & 0.39 & 0.31 & 0.47 & 7.35 & -0.87 \\
\hline
\end{tabular}

These ARMA processes are not sufficient to make long term predictions as they converge to their mean value. Therefore, for the case of day ahead forecast, they are replaced by SARIMA models with 24 hours seasonality.

\section{B. Results}

The HMMs are chosen to have 10 and 8 states for up and down regulation respectively while the number of the Gaussian mixtures is set to 4. Tables IV and V show the MAE and SMAPE scores of the BM volume and premium forecasts for both the HMM and the reference model and both one hour and day ahead forecasts. The two methods achieve quite similar scores for one hour ahead forecasts. It is also clear that up regulation volumes and premiums are better predictable than down regulation counterparts. For the case of day ahead forecasts the scores are quite poor for both models.

TABLE IV

AVERAGE HOURLY MAE/SMAPE SCORES FOR VOLUME FORECASTING

\begin{tabular}{|c|c|c|c|}
\cline { 3 - 4 } \multicolumn{2}{c|}{} & MAE (Up/Down) & SMAPE (Up/Down) \\
\hline \multirow{2}{*}{ 亲 } & $1 \mathrm{~h}$ & $14.3 / 27.2$ & $0.43 /-0.35$ \\
\cline { 2 - 4 } & $12-36 \mathrm{~h}$ & $21.4 / 45.2$ & $0.75 /-0.88$ \\
\hline \multirow{2}{*}{$\mathrm{s}$} & $1 \mathrm{~h}$ & $15.2 / 28.1$ & $0.49 /-0.40$ \\
\cline { 2 - 4 } & $12-36 \mathrm{~h}$ & $20.1 / 43.1$ & $0.73 /-0.82$ \\
\hline
\end{tabular}

TABLE $\mathrm{V}$

\begin{tabular}{|c|c|c|c|}
\hline & MAE (Up/Down) & SMAPE (Up/Down) \\
\hline $\bar{\Sigma}$ & $1 \mathrm{~h}$ & $0.74 / 1.18$ & $0.41 /-0.31$ \\
\hline ¿्丶 & $12-36 \mathrm{~h}$ & $1.2 / 2.0$ & $0.61 /-0.78$ \\
\hline 3 & $1 \mathrm{~h}$ & $0.7 / 1.12$ & $0.42 /-0.31$ \\
\hline$\frac{2}{4}$ & $12-36 \mathrm{~h}$ & $1.4 / 2.1$ & $0.70 /-0.81$ \\
\hline
\end{tabular}

AVERAGE HOURLY MAE/SMAPE SCORES FOR PREMIUM FORECASTING

In $\mathrm{BM}$ forecasting it is very important to predict the balancing state correctly. Because of the floor/cap price in the spot market, the state prediction becomes even more important 
than the price value itself. Table VI shows the percentage of the states that have been correctly predicted. The proposed HMM method outperforms the reference model for both the one hour ahead and day ahead forecasts. In the first case, it manages to predict correctly approx. $75 \%$ of the states while in the second case the score is approx. $45 \%$.

TABLE VI

CORRECT STATE PREDICTION SCORE

\begin{tabular}{|c|c|c|}
\cline { 2 - 3 } \multicolumn{1}{c|}{} & 1 & $12-36$ \\
\hline HMM & $73 \%$ & $44 \%$ \\
\hline ARMA & $60 \%$ & $38 \%$ \\
\hline
\end{tabular}

Fig. 6 shows the one hour ahead volume prediction for both the HMM and the reference model. We see that the HMM predicts the BM states quite well. On the other hand the ARMA model is better predicting the values, specially the peaks. The same is concluded by the BM premium predictions in fig. 7. This is the reason why although the HMM method outperforms in state prediction, the MAE and SMAPE scores are similar.

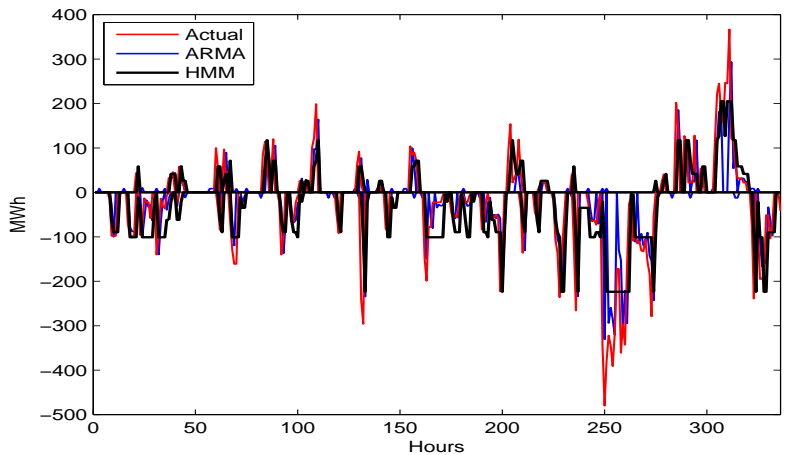

Fig. 6. One hour ahead BM volume predictions

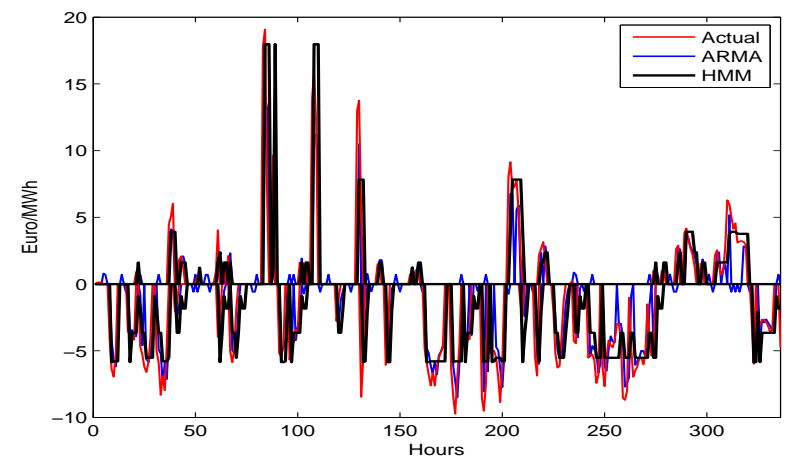

Fig. 7. One hour ahead BM premium predictions

\section{CONCLUSION}

A HMM based method is proposed in this paper for BM forecasting. This method provides forecasts for both the BM premiums and the BM volumes with mixed results. The one hour ahead forecasts are achieving good results which are similar to other methods. The day ahead results cannot manage to capture the BM movement so well. This also applies to the reference model and it is in agreement with the results in [8] where all the models fail to give good results for 12-36 hours ahead forecasts. The reason for this is that the BM states are quite random without presenting some seasonal pattern. Further research is needed in order to identify factors that influence the BM prices. Such explanatory variables can be for example the wind and solar power production or the load.

\section{ACKNOWLEDGEMENT}

This work was sponsored by SweGRIDS, the Swedish Centre for Smart Grids and Energy Storage, www.swegrids.se.

\section{REFERENCES}

[1] EuropeanCommission, "Energy for the future: Renewable sources of energy. White paper for a community strategy and action plan," 1997.

[2] R. Weron, "Electricity price forecasting: A review of the state-of-theart with a look into the future," International Journal of Forecasting, vol. 30, no. 4, pp. 1030-1081, Oct. 2014.

[3] L. Mordasiewicz, "Price forecasting in the balancing mechanism," Rynek Energii, pp. 153-160, 2011.

[4] S. Jaehnert, H. Farahmand, and G. L. Doorman, "Modelling of prices using the volume in the Norwegian regulating power market," in PowerTech, 2009 IEEE Bucharest. IEEE, 2009, pp. 1-7.

[5] M. Olsson and L. Soder, "Generation of regulating power price scenarios," in 2004 International Conference on Probabilistic Methods Applied to Power Systems, 2004, pp. 26-31.

[6] M. Olsson and L. Söder, "Modeling real-time balancing power market prices using combined SARIMA and Markov processes," IEEE Transactions on Power Systems, vol. 23, no. 2, pp. 443-450, 2008.

[7] M. O. Brolin and L. Söder, "Modeling Swedish real-time balancing power prices using nonlinear time series models," in Probabilistic Methods Applied to Power Systems (PMAPS), 2010 IEEE 11th International Conference on. IEEE, 2010, pp. 358-363.

[8] G. Klæboe, A. L. Eriksrud, and S.-E. Fleten, "Benchmarking time series based forecasting models for electricity balancing market prices," Energy Systems, vol. 6, no. 1, pp. 43-61, Mar. 2015.

[9] L. Rabiner, "A tutorial on hidden Markov models and selected applications in speech recognition," Proceedings of the IEEE, vol. 77, no. 2, pp. 257-286, 1989.

[10] S. R. Eddy, "What is a hidden Markov model?" Nature Biotechnology, vol. 22, no. 10, pp. 1315-1316, Oct. 2004

[11] M. R. Hassan and B. Nath, "Stock market forecasting using hidden Markov model: a new approach," in Intelligent Systems Design and Applications, 2005. ISDA'05. Proceedings. 5th International Conference on, 2005, pp. 192-196.

[12] A. Gupta and B. Dhingra, "Stock Market Prediction Using Hidden Markov Models," in Engineering and Systems (SCES), 2012 Students Conference on, 2012, pp. 1-4.

[13] J. Zhang, J. Wang, R. Wang, and G. Hou, "Forecasting next-day electricity prices with Hidden Markov Models," in Industrial Electronics and Applications (ICIEA), 2010 the 5th IEEE Conference on, 2010, pp. $1736-1740$.

[14] A. Gonzalez, A. Roque, and J. Garcia-Gonzalez, "Modeling and forecasting electricity prices with input/output hidden Markov models," IEEE Transactions on Power Systems, vol. 20, no. 1, pp. 13-24, 2005.

[15] G. L. Kouemou, "History and theoretical basics of hidden Markov models," Hidden Markov Models, theory and applications',(Intech Pub, 2011) pp, pp. 3-24.

[16] ENTSO-E, "Final Report on Operational Reserves," Tech. Rep., May 2012.

[17] "Market data I Nord Pool." [Online]. Available: http://www.nordpoolspot.com/Market-data1/Regulating-Power1/ Regulating-Power--Area1/SE2/Norway/?view=table

[18] A. L. Eriksrud and J. Braathen, "Spot Market Bidding Taking the Balancing Power Market Into Account," Ph.D. dissertation, 2012. 